\title{
La place du jeu dans les loisirs et l'animation enfance et jeunesse : représentations des acteurs
}

The role of play in leisure activities for children and young people: actors' representations

\section{Stéphanie Constans, Véronique Rouyer et Emmanuèle Gardair}

\section{(2) OpenEdition Journals}

Édition électronique

URL : https://journals.openedition.org/sdj/2028

DOI : $10.4000 /$ sdj.2028

ISSN : 2269-2657

\section{Éditeur}

Laboratoire EXPERICE - Centre de Recherche Interuniversitaire Expérience Ressources Culturelles Education

\section{Référence électronique}

Stéphanie Constans, Véronique Rouyer et Emmanuèle Gardair, «La place du jeu dans les loisirs et l'animation enfance et jeunesse : représentations des acteurs », Sciences du jeu [En ligne], 12 | 2019, mis en ligne le 15 décembre 2019, consulté le 12 décembre 2022. URL : http:// journals.openedition.org/sdj/2028; DOI : https://doi.org/10.4000/sdj.2028

Ce document a été généré automatiquement le 12 décembre 2022.

\section{(c) (i) (9)}

Creative Commons - Attribution - Pas d'Utilisation Commerciale - Pas de Modification 4.0 International - CC BY-NC-ND 4.0

https://creativecommons.org/licenses/by-nc-nd/4.0/ 


\section{La place du jeu dans les loisirs et l'animation enfance et jeunesse : représentations des acteurs}

The role of play in leisure activities for children and young people: actors' representations

Stéphanie Constans, Véronique Rouyer et Emmanuèle Gardair

\section{Le jeu et les loisirs organisés en questions}

1 Les loisirs, et en particulier les loisirs organisés dans le champ de l'animation enfance et jeunesse, restent peu étudiés en France en psychologie du développement. Ces pratiques sont davantage examinées en sociologie et en sciences de l'éducation. Par contre, elles font l'objet de nombreux travaux dans la communauté internationale qui étudient les liens entre les pratiques de loisirs et les dimensions sociales et cognitives du développement chez l'enfant et l'adolescent-e (Bartko et Eccles, 2003 ; Fredricks et Eccles, 2008 ; Mahoney et al., 2005 ; Vandell et al., 2015). À l'instar de l'école et de la famille, les loisirs participent aux processus d'éducation et de socialisation des enfants et des adolescent-e-s et contribuent à leur développement (Bronfenbrenner, 1979; Leversen et al., 2012). La participation à des activités de loisir permet aux enfants et aux adolescent-e-s de faire des choix et d'élaborer des projets. En lien avec leurs intérêts et leurs besoins psychologiques (Kindelberger, Le Floc'h et Clarisse, 2007), ces pratiques contribuent à l'épanouissement, l'expression et la réalisation de soi. Elles peuvent se réaliser seul ou à plusieurs dans le cadre de différentes structures d'animation relevant du secteur associatif, de fédérations, d'équipements ou de dispositifs initiés par l'État et les collectivités locales (centre de loisirs, espace jeunes, maison de quartier, centre social, maison de la jeunesse et de la culture, etc.) (Augustin et Gillet, 2000) ou de lieux moins structurés (à la maison, en ville, etc.). Enfin, ces pratiques recouvrent différents domaines : sportif, artistique, culturel ou social (Boyer, Bounoure et Delclaux, 1991). Passmore et French (2001) en proposent une classification en fonction du but poursuivi 
dans l'activité de loisir : des activités de réalisation de soi demandant des régulations cognitives et sociales (arts, sports, etc.), des activités sociales tournées vers la relation avec les pairs (discuter, bavarder, sortir, etc.) et des activités de détente sans exigences particulières (farniente, regarder la télévision, écouter la radio, etc.). Le jeu n'apparaît pas spécifiquement dans ces analyses, alors même qu'il est une forme de loisir, sans doute la forme originaire puisque particulièrement présent chez l'enfant (Brougère, 2005). On peut alors se demander où se situe le jeu dans ces pratiques, en particulier dans les loisirs organisés dans le champ de l'animation enfance et jeunesse? Quelle place lui accordent les adultes encadrant ces pratiques, mais également les enfants et les adolescent-e-s dans leurs loisirs?

2 Cet article se propose de répondre à cette question en examinant, sous ce nouvel angle qu'est la place du jeu dans les loisirs organisés, les résultats de nos travaux sur les représentations des loisirs, d'une part des professionnel-le-s exerçant dans des structures d'animation enfance et jeunesse, d'autre part des enfants usagers d'accueils de loisirs sans hébergement et des adolescent-e-s fréquentant des espaces jeunes. En effet, nous ne pouvons envisager de comprendre les représentations et les pratiques des animateurs-trices sans examiner les intérêts et les positionnements des enfants et des adolescent-e-s eux-mêmes. S'il est vrai que les enfants et les adolescent-e-s agissent dans un champ structuré par les adultes, ils et elles n'en investissent pas moins leur propre espace d'autonomie et leurs propres projets (Barrère, 2011 ; Delalande, 2001).

\section{Différentes formalisations des loisirs organisés}

3 Issue de l'éducation populaire, l'animation enfance et jeunesse représente une forme d'éducation particulière, s'inspirant de l'éducation nouvelle, construite sur une double distinction avec l'école. Une première distinction concerne les contenus d'apprentissages : l'animation se propose de dépasser

les savoirs et connaissances disciplinaires de l'école pour développer l'ensemble des composantes du développement physique, psychique et social de l'enfant [...], les actions éducatives sont ainsi essentiellement orientées vers les compétences, les savoir-faire et les savoir-être qui permettront au sujet adulte de trouver sa place sociale (Roucous, 2007, p. 68).

Une autre distinction renvoie aux méthodes pédagogiques : l'animation privilégie des méthodes participatives, autrement dit des méthodes engageant l'individu et lui permettant d'acquérir des contenus par la pratique, dans un rapport faisant sens pour lui (Roucous, 2007). Toutefois, certains auteurs pointent le fait que cette distinction n'est pas aussi marquée et explicite. Houssaye (1998) montre par exemple que les centres de vacances et de loisirs des enfants restent " prisonniers de la forme scolaire ", tant par la programmation que par la gestion temporelle et l'organisation spatiale des activités. De son côté, Roucous (2007) précise que la prise en charge des enfants dans les structures d'animation se construit autour d'un projet éducatif fixant les ambitions en matière d'évolution de l'enfant. Dès lors que l'enfant est pris en charge, une attention permanente à son évolution, au développement de ses compétences et performances est portée par l'institution (quelle qu'elle soit). Haglund (2015), dans le cadre de centres de loisirs en Suède, relève un décalage entre le choix de l'enfant et celui de l'adulte pour l'enfant. L'auteur recense les activités libres, choisies par l'enfant, les opposant aux activités thématiques, choisies et pensées pour les éveiller, voire les éduquer, par les animateurs-trices. Ces dernier-e-s ressentent une tension entre leur volonté de 
laisser la liberté aux enfants de participer aux activités et leur souhait de stimuler l'intérêt des enfants et de les encourager dans des activités pour lesquelles ils sont a priori réticents.

Dans une analyse de l'usage de la notion d'informel dans le champ de l'éducation, Brougère et Bézille (2007) mettent en exergue le fait que dès qu'une dimension éducative est perçue, le désir de renforcer ses effets conduit à certaines formalisations. En référence à cette analyse, Roucous (2007) distingue trois formes d'éducation dans le champ de l'animation sur un continuum renvoyant au degré de formalisation. Une première forme, plutôt scolaire, consiste à organiser la transmission de savoirs dans une relation pédagogique unilatérale et hiérarchique. Une autre forme éducative de l'animation, fondée sur la participation active, mais conditionnant le loisir à un résultat pour ce qui touche à l'apprentissage et au développement, amène l'animateur-trice à une forme de gouvernance et à une élaboration, qui préside à l'activité de loisir. Enfin, une forme d'éducation informelle, caractéristique notamment des ludothèques, est pensée " comme la mise en œuvre réfléchie, construite et organisée de l'informel » et consiste "à installer un cadre spatial, temporel et réglementaire qui rend possible mais aussi qui organise cette activité de jeu et de loisir en donnant des modalités, des formes, voire des règles » (Roucous, 2007, p. 71).

Partant de ces analyses, il n'est pas aisé d'évoquer la question du jeu et de sa place dans le cadre des loisirs organisés des enfants et des adolescent-e-s dans le champ de l'animation, si l'on considère que le jeu, et plus largement le loisir, est un temps libre, non contraint, géré par l'enfant ou l'adolescent-e lui-même, échappant à l'initiative de l'adulte. Cette difficulté tient d'une part, comme vu ci-avant, à l'inscription des structures qui encadrent les loisirs des enfants et des adolescent-e-s dans une perspective (le plus souvent) éducative valorisant une forme contraignante de pratiques de loisirs (Roucous, 2007) et d'autre part, à la fréquentation plus ou moins contrainte et non choisie des enfants dans ces structures (Constans et Gardair, 2018); l'enfant devant alors trouver sa place dans des temps organisés pour lui par les adultes. Mollo-Bouvier (1994, p. 81), dans une analyse sociologique du temps libre de l'enfant, montre que celui-ci, organisé principalement à partir du temps de travail des parents, se présente toujours comme "un temps bien rempli à l'avance», "un temps programmé, encadré, occupé ». Dans une étude traitant des effets du temps scolaire, des loisirs et du travail parental sur les rythmes d'attention de l'enfant, Le Floc' $\mathrm{h}$ (2005) confirme ce constat et précise que les activités de loisirs pratiquées par les enfants répondent davantage au temps des parents, en matière de disponibilités et d'exigences professionnelles, qu'au temps des enfants et font ainsi rarement écho à un choix de leur part. Dans ce contexte, nous pouvons nous poser la question du pouvoir de décision de l'enfant, indissociable de l'idée de loisir, comme le montre Houssaye (1995) au sein des accueils de loisirs. En effet, « une activité ludique et plus largement de loisir ne trouve et ne conserve toute sa forme que dans la mesure où le sujet décide de son activité. Il n'y a de jeu ou de loisir que tant que l'acteur le construit et le pense comme tel » (Roucous, 2007, p. 64).

\section{Le jeu : une affaire de sens}

7 La définition du jeu est un exercice complexe tant ce terme recouvre une diversité d'activités et d'usages langagiers (Brougère, 1995, 2005 ; Lillard, 2015 ; Winnykamen, 
2009). Néanmoins, Brougère $(2005$, p. 7$)$ montre qu'il est possible de dégager «les principes qui organisent l'usage du terme " et "de mettre en évidence plusieurs niveaux de réalité pouvant, en français tout au moins, recevoir le vocable jeu ». Ainsi, le jeu peut désigner : un objet (matériel de jeu) ${ }^{1}$; une structure, un ensemble de règles et de principes (existant indépendamment des joueurs) ; une activité (ludique), liée au fait de jouer (Brougère, 1995). C'est à ce troisième niveau que nous nous intéressons ici.

La polysémie de la notion est d'autant plus grande qu'il échappe à l'appropriation exclusive par une discipline et que les théories qui lui sont consacrées abondent. En psychologie du développement, les auteurs ont principalement conceptualisé le rôle du jeu dans le cadre du développement de l'enfant (Winnykamen, 2009). La plupart des approches (à l'instar de celle de Piaget) ont défini le jeu selon des catégories de comportements qui suivent ou marquent la progression du développement de l'enfant (jeu fonctionnel, jeu de construction, jeu de faire-semblant, jeu de règles). D'autres approches recensées par Lillard (2015) ont davantage insisté sur la disposition fonctionnelle à partir de laquelle les activités ludiques se développent. Sont ainsi pointés par l'auteur comme caractéristiques $\mathrm{du}$ jeu: un comportement intrinsèquement motivé (Berlyne, 1960), une fin plus que des moyens (Bruner, 1972), une expérience subjective qui renvoie à « ce que je peux faire avec cet objet, plutôt que ce que cet objet peut faire » (Hutt et al., 1989), ou encore le fait d'avoir une dimension de « comme si ». Pour Wallon (1968, p. 58), le nom de jeu a été donné à des activités diverses "par assimilation à ce qu'est le jeu chez l'adulte»: le jeu est «d'abord délassement et, par-là, s'oppose à l'activité sérieuse qu'est le travail. Mais ce contraste ne peut exister chez l'enfant qui ne travaille pas encore et dont le jeu est toute l'activité». Wallon précise cependant que le jeu recouvre toute l'activité de l'enfant»tant qu'elle reste spontanée et ne reçoit pas ses objets des disciplines éducatives » (Wallon, 1968, p. 57).

9 La pluralité des approches théoriques, développées dans les différentes disciplines et au sein même d'une discipline, nous invite à la prudence quant à la définition du jeu. Dans cette perspective, Brougère (2005, p. 42) recommande «non pas de délimiter le jeu par distinction, mais de proposer des points de repère afin de disposer de caractéristiques du jeu, en concédant qu'elles puissent être plus ou moins présentes ». Au regard de la contribution de différents auteurs à la connaissance du jeu, Brougère $(2005,2012)$ propose cinq caractéristiques du jeu: 1 ) le second degré (caractère de faire semblant), 2) la prise de décision (succession de décisions qui organisent, construisent et transforment l'activité), 3) la règle (implicite ou explicite, codifiée ou inventée, négociable et modifiable), 4) la frivolité (absence de finalité du jeu, mise à distance des conséquences) et 5) l'incertitude (de l'issue et du déroulement du jeu, qui découle des choix du joueur). Ces différentes caractéristiques permettent «de comprendre les logiques des activités qui peuvent être dénommées "jeu" ou qui sans l'être peuvent en être proches » (Brougère, 2005, p. 59). Cette approche met en évidence la variété et la malléabilité du jeu dont les contours et les frontières apparaissent flous et, par-là, « combien le jeu est affaire de sens donné à la situation » (Brougère, 2005, p. 61).

Dans cette perspective, et en référence à Yonnet $(1999$, p. 77$)$ qui considère que le loisir renvoie à « la possibilité ou [à] la permission de faire ce que l'on veut, une fois achevé ou suspendu le faire-obligé ${ }^{2}$ ou le faire-contraint ${ }^{3}$ ", Brougère (2005) souligne la nécessité de rétablir le lien du jeu avec le loisir. De la même façon que le jeu, le loisir se définit par le sens donné à l'(in)activité, les raisons pour lesquelles on s'y livre. Selon 
Brougère (2005, p. 137), le jeu apparaît comme une forme de loisir : «la spécificité du jeu au sein du loisir ne serait pas de l'ordre d'une catégorisation étroite (le jeu comme un type de loisir parmi d'autres), mais de l'ordre d'une forme, d'une dimension supposant l'interactivité, l'implication du joueur dans la décision, facteur d'incertitude ». Ainsi, le loisir devient ludique » quand, au-delà du second degré et de l'absence de risque, la participation se fait active, interactive, l'action du participant influant le devenir de l'action, même si cette influence est en partie illusoire " (Brougère, 2005, p. 137).

\section{Loisirs et socialisation de genre}

11 Par ailleurs, un certain nombre de travaux indiquent que durant l'enfance comme à l'adolescence, les filles et les garçons s'impliquent différemment dans les activités de loisirs: si les premières privilégient les activités créatives et culturelles (danse, musique, etc.), les seconds s'engagent davantage dans les activités sportives (Arthenstaedt, Mikula et Bredt, 2009 ; Bradley et Inglis, 2012 ; McHale et al., 2004 ; Sharp et al., 2007 ; Simpkins et al., 2005). Plus précisément, les activités sportives semblent constituer encore aujourd'hui un espace "masculin» (Davisse et Louveau, 1998), au regard du nombre toujours plus important de garçons et d'hommes dans le domaine sportif (licenciés d'un club, entraineurs, étudiants en STAPS, conseillers techniques ou journalistes) (Raibaud, 2014). De plus, des différences dans le type et la fréquence des activités sportives persistent: certaines activités étant qualifiées de "féminines" (danse, gymnastique, etc.), d'autres de «masculines» (football, rugby, boxe, etc.). Des travaux montrent également un décrochage d'une pratique sportive encadrée chez les filles durant l'adolescence (Davisse et Louveau, 1998; Maruejouls, 2011). Ainsi, les activités sportives contribuent au processus de socialisation de genre et à la construction des identités sexuées de l'enfance à l'âge adulte, à travers notamment la valorisation de certains aspects liés à la masculinité (force physique, esprit de compétition, affirmation de soi, etc.) et de la féminité (recherche de l'esthétique par un modelage de l'apparence) et le travail du corps sexué (Court, 2010; Davisse, 2006; Mercier-Lefèvre, 2014).

Du côté des professionnels des structures de loisirs, des analyses soulignent d'autres aspects du processus de socialisation de genre à l'œuvre dans les activités de loisirs : par exemple, l'offre des activités de loisirs à destination des usagers-ères, les attentes genrées des adultes quant aux activités de loisirs, la présence des professionnel-le-s dans les structures de loisirs et la façon dont évoluent leurs carrières en termes de fonctions et de prises de responsabilités (Bacou, 2010 ; Bessin, 2009 ; Gillet et Raibaud, 2006 ; Herman, 2007 ; Maruéjouls, 2011 ; Raibaud, 2007 ; Smith et al., 2012). Ainsi, cet espace des loisirs n'échappe pas à la problématique de la socialisation de genre et de la construction identitaire (Rouyer, 2007 ; Rouyer, Croity-Belz et Prêteur, 2010 ; Rouyer, Mieyaa et Le Blanc, 2014).

En lien avec cette question de la place du jeu aujourd'hui dans les loisirs des enfants et des adolescent-e-s, on se demande plus spécifiquement dans cet article si cette référence à la dimension ludique est présente dans les représentations des loisirs des animateurs-trices, des enfants et des adolescent-e-s, et ce de façon différenciée selon le genre. 


\section{Terrains et dispositif d'enquête}

14 Nous avons conduit deux études sur les représentations et les pratiques des loisirs dans le champ de l'animation enfance et jeunesse. Une première étude (Gardair et Constans, 2011) interroge les représentations et les pratiques des animateurs-trices, une seconde étude les représentations et les pratiques des enfants et des adolescent-e-s (Constans et Gardair, 2018).

\section{Une enquête auprès des professionnel-le-s et des enfants et adolescent-e-s}

Dans un premier temps, nous avons recueilli des données sur les représentations et les pratiques de 80 animateurs-trices (36 hommes et 44 femmes) exerçant dans diverses structures d'animation (accueils de loisirs sans hébergement, centres socioculturels, maisons de quartiers, espaces jeunes, maisons des jeunes et de la culture, associations sportives ou culturelles) dans les régions de Bretagne et de Pays de la Loire. 46 interviennent auprès d'un public enfant ( 16 hommes et 30 femmes), 34 auprès d'un public adolescent (20 hommes et 14 femmes). Dans cet échantillon, nous relevons une répartition inégale des professionnel-le-s dans l'encadrement des publics enfant et adolescent, qui fait écho au processus de féminisation des métiers de l'animation et plus particulièrement aux représentations sexuées des "qualités naturelles" des femmes considérées plus compétentes pour intervenir auprès de jeunes publics, notamment dans des activités d'« attention envers autrui, au soin, à l'affection et à la proximité », qu'Herman appelle le care (Herman, 2007, p. 122).

Les animateurs-trices ont été interrogé-e-s par le biais d'entretiens semi-directifs sur les thématiques suivantes : la définition et le développement de l'enfant/adolescent, les méthodes pédagogiques et les pratiques de loisirs susceptibles de favoriser le développement, les pratiques éducatives effectives et les activités de loisirs proposées. Les participant-e-s ont été sollicité-e-s dans le cadre d'une passation individuelle au sein de leur structure sur un temps libéré sans la présence du public. La durée moyenne de l'entretien était de trente minutes; celui-ci était enregistré, puis retranscrit intégralement.

Dans un second temps, nous avons recueilli des données sur les représentations et les pratiques de 50 enfants et adolescent-e-s : 31 enfants (16 garçons et 15 filles) usagers d'un accueil de loisirs sans hébergement de l'agglomération nantaise, âgé-e-s de 5 à 10 ans $^{4} ; 19$ adolescents (14 garçons et 5 filles), fréquentant un espace jeunes de l'agglomération rennaise, âgé-e-s de 11 à 18 ans $^{5}$. Les deux structures de loisirs sont situées dans un environnement périurbain et les deux communes présentent les mêmes caractéristiques quant au nombre d'habitants (moins de 6000 habitants) et au niveau socio-économique (CSP mixte, taux d'activité de $76 \%$ des 15 à 64 ans) $)^{6}$. La faible présence des filles dans notre échantillon d'adolescent-e-s illustre le constat général de l'hégémonie de la présence des garçons dans les loisirs organisés et de la disparition des filles des équipements et des structures de loisirs, ces dernières se repliant vers la sphère privée (Maruéjouls, 2011).

18 Les enfants et les adolescent-e-s ont été interrogé-e-s au moyen d'entretiens semidirectifs sur les thématiques suivantes: la définition et la fonction des loisirs, la définition et la fonction de ceux qui les encadrent, les pratiques de loisirs effectives et 
souhaitées. Tous les participant-e-s ont été volontaires pour participer à cette étude (après accord de leurs parents pour les mineur-e-s). En accord avec le responsable, nous avons rencontré les enfants et les adolescent-e-s individuellement au sein de la structure sur un temps libéré à l'écart du groupe. La durée moyenne de l'entretien était de vingt minutes ; celui-ci était enregistré, puis retranscrit intégralement.

\section{Une analyse lexicométrique des données d'entretiens}

Nous avons réalisé des analyses lexicales sur les différents corpus recueillis à l'aide du logiciel Alceste (Reinert, 1983, 1993), qui est un outil d'aide à l'interprétation de corpus textuels présentant une certaine homogénéité et un volume minimum. L'objectif est d'obtenir un classement statistique des phrases du corpus, en fonction de la distribution des mots (unités de contexte élémentaires ou UCE), permettant de dégager les principaux mondes lexicaux (ou classes) et d'identifier des répertoires caractéristiques du discours de l'ensemble des participants (corpus). Alceste établit une classification hiérarchique descendante (CHD) qui consiste à répartir l'ensemble des sujets en classes, en fonction de la ressemblance de leur profil par rapport à l'ensemble des indicateurs utilisés. Le logiciel associe ensuite les variables illustratives aux classes, ce qui n'exclut pas une certaine variabilité des sujets réels au sein de chaque profil ou classe. L'anti-profil complète chaque profil par les traits les plus antinomiques, qui contribuent à identifier les attributs les plus opposés à chaque profil. Alceste élabore ainsi une modélisation du corpus, le reconstruit en classes d'énoncés qui représentent des profils différents et qui sont considérées par Reinert (1993) comme le reflet des représentations du sujet, chacune d'elles renvoyant à la manière dont celui-ci appréhende un objet en fonction de sa propre identité et de son intention. Ces classes doivent faire l'objet de la part du chercheur d'une interprétation qui dépend des objectifs de l'analyse.

Concernant les discours des animateurs-trices, une première CHD a été réalisée sur le corpus global (Gardair et Constans, 2011), qui montre que les variables sexe et âge du public accueilli discriminent partiellement les classes, mais que, à l'inverse, la variable type de structure n'illustre pas de classe. Une CHD sur le corpus des animateurs et une autre sur celui des animatrices confirment et permettent de compléter et préciser ces premiers résultats.

21 S'agissant du discours des enfants et des adolescent-e-s, une seule CHD sur le corpus global a été réalisée en raison de l'effectif. Elle montre que la variable âge discrimine les classes, la variable sexe étant partiellement associée. Un tri croisé sur la variable sexe permet de compléter cette analyse et de distinguer le discours des enfants et des adolescent-e-s selon leur sexe.

\section{Des représentations du loisir distinctes selon le genre des professionnel-le-s}

\section{Des représentations des animateurs orientées vers l'accompagnement éducatif des adolescent-e-s}

La CHD réalisée sur le corpus des animateurs a permis de dégager quatre profils différenciés (classes) à partir de leurs discours. Elle met en exergue des pratiques 
tournées majoritairement vers le public des adolescent-e-s puisqu'une seule représentation (classe 1, $25 \%$ du discours, 523 UCE) est caractéristique des loisirs destinés aux enfants. De façon spécifique pour ce public enfant, les animateurs inscrivent les loisirs dans une perspective éducative et envisagent des formes de pratiques de loisirs organisées et contraignantes. «Le centre de loisirs est une structure éducative au même titre que l'école, sauf qu'on n'apporte pas les mêmes choses, mais on participe à la construction de l'enfant ». Ils considèrent que les loisirs ont un rôle essentiel dans le développement de l'enfant, complémentaire de celui de l'école et de la famille, et qu'ils favorisent des formes d'apprentissages et contribuent au processus de socialisation de l'enfant. « Les structures de loisirs peuvent être un moyen de socialiser l'enfant ». Le terme "jeu » est absent du discours de ces animateurs. Même si cette forme d'animation valorise des méthodes participatives, elle conditionne le loisir à un résultat en termes d'apprentissage et de développement et amène l'animateur à une

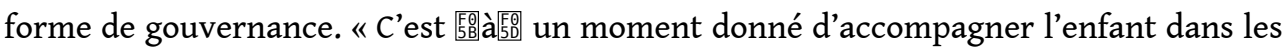
apprentissages qu'il ne peut avoir, ni dans sa famille, ni à l'école, ce sont des apprentissages complémentaires ».

Les trois autres représentations (classes 2, 3 et 4 ) des animateurs sont davantage caractéristiques des loisirs destinés aux adolescent-e-s. Une première représentation (classe 2, $22 \%$ du discours, 456 UCE) envisage les loisirs pour ce public comme un espace de pratiques ou d'activités, plutôt physiques et culturelles, relevant du domaine marchand. « Ils nous demandent de les emmener au karting, faire de la moto, du paintball ». Elles s'inscrivent dans un programme élaboré par l'animateur, en concertation avec les adolescent-e-s et parfois autofinancées par ces dernier-e-s. "Les jeunes doivent chercher un camping, le stage de moto, savoir combien ça va coûter et proposer des solutions d'autofinancement». Certaines de ces activités peuvent être caractérisées comme des activités ludiques au regard des dimensions du jeu développées par Brougère (2005). Toutefois, le terme "jeu» n'apparaît pas dans le discours des animateurs. Par ailleurs, ces activités semblent plutôt correspondre aux goûts majoritaires des garçons.

Une deuxième représentation (classe 3, $19 \%$ du discours, 384 UCE) appréhende les loisirs comme un espace de socialisation et d'éducation à la citoyenneté, privilégiant l'ouverture des adolescent-e-s sur l'extérieur et leur implication dans la cité. "Leur proposer en même temps un lieu d'accueil et des activités qui les intéressent, qui les ouvrent vers l'extérieur »; "Ce qu'on met en avant c'est l'implication du jeune dans la vie de la cité, c'est plus la citoyenneté ». Les activités sont proposées par le biais de projets ou d'activités à nouveau plutôt sportives et culturelles. « Ils aimeraient faire un projet, monter un festival de danse hip hop, des trucs comme ça »; « Essayer de voir ce qui suscite l'intérêt chez le jeune, si on voit que c'est plus le sport, la culture, c'est à toi de surfer sur cette accroche-là ». Comme précédemment, ces actions semblent davantage être celles que plébiscitent les garçons. «On propose différentes actions de l'accueil formel sportif à l'accueil informel ».

Enfin, une troisième représentation des animateurs (classe 4, $34 \%$ du discours, 701 UCE) dessine une catégorisation des loisirs à l'adolescence en fonction de l'âge et des aspects spécifiques du développement dont il est nécessaire de tenir compte dans la proposition et la mise en place d'activités.

C'est dur de faire rentrer l'adolescent dans un cadre puisque l'adolescence est à la fois une période de transformations et de changements que ce soit physique ou psychologique. 
L'adolescence pour moi, c'est une période de transition, la découverte de la société, du monde adulte. masculins, que ces dernières évoquent davantage des pratiques de loisirs en direction des enfants, ce qui fait écho aux analyses de genre concernant ce secteur professionnel. Deux représentations (classes 1 et 2 ) sont caractéristiques des loisirs dédiés aux enfants; elles sont (logiquement) davantage développées par des animatrices intervenant auprès de ce public. Une première représentation (classe $1,25 \%$ du discours, 536 UCE) valorise les dimensions affective et sociale dans les loisirs, mettant en exergue la primauté des aspects relationnels (écoute, sécurité, etc.) dans les interactions avec les enfants. «On fait des bases de communication tout de suite parce qu'on intervient beaucoup sur la communication » « Tout en leur inculquant aussi de la sécurité ». Dans cette représentation aussi le jeu est valorisé, mais de façon moindre. «Les amuser, leur apporter un côté affectif qu'ils peuvent aussi rechercher ». L'aspect relationnel et le jeu priment sur la mise en œuvre d'activités particulières qui seraient propices au développement de l'enfant, ce que confirme l'anti-profil qui écarte la programmation d'activités pour prendre en compte et servir les différentes étapes du développement de l'enfant. «J'ai jamais eu autant de satisfaction que de voir un gamin avec le sourire ".

29 Une deuxième représentation (classe 2, $35 \%$ du discours, 732 UCE) est davantage centrée sur le développement de l'enfant et c'est par rapport à celui-ci que sont organisés les loisirs des enfants par le biais de différentes activités à visée éducative. Les animatrices considèrent ici que leur rôle est d'accompagner les enfants dans leur développement afin de les conduire vers l'autonomie. De la même façon que les animateurs (classe 4, CHD corpus animateurs), elles définissent le développement de l'enfant en termes de catégories d'âges et appréhendent ainsi les loisirs en fonction de cette catégorisation. "C'est à l'adulte de faire des activités adaptées en fonction justement du stade de développement de l'enfant». Toutefois, elles appréhendent également l'enfant plus individuellement et en pensant le développement dans sa globalité, au-delà de la seule catégorisation en termes d'âge. «Il faut toujours adapter effectivement en fonction de la tranche d'âge, mais surtout en fonction du développement personnel de chaque enfant "; «Les enfants, tout comme les adultes, progressent tout au long de leur vie ».

Ces deux représentations laissent apparaitre chez les animatrices une distinction entre, d'une part, des activités de care couplées aux activités ludiques et, d'autre part, des 
activités pédagogiques qui seraient sources d'apprentissages et de développement. La primauté des dimensions affective et sociale dans les loisirs s'illustre par ailleurs au niveau des pratiques des animatrices en direction du public des adolescent-e-s qui apparaissent dans une troisième représentation (classe 3, $20 \%$ du discours, 428 UCE) ${ }^{7}$. "Personne chez eux ne leur demande s'ils vont bien et s'ils ont passé une bonne journée, nous on le fait quand ils rentrent des cours et qu'ils passent par le centre ». Même si ces dimensions s'actualisent différemment au regard du niveau de développement de ces dernier-e-s, les animatrices développent une représentation soulignant une finalité de convivialité et de détente entre pairs, centrée sur l'écoute et l'expression et autour d'activités informelles et ludiques comme jouer au babyfoot/ billard, écouter de la musique, jouer sur l'ordinateur, la console ou autres supports numériques, etc. "Ils sont de moins en moins dans la demande d'activités en tant que telles »; " Ici il y a un accueil un peu plus informel, ils ont envie de faire une partie de babyfoot ». Si les animatrices soulignent, de la même façon que les animateurs (classe 3, CHD corpus animateurs), la dimension socialisante des loisirs à l'adolescence, elles ne l'envisagent pas dans une perspective éducative visant précisément l'apprentissage de règles sociales ou la formation du futur citoyen.

Par ailleurs, les animatrices distinguent auprès de ce public adolescent les activités à destination des filles et celles à destination des garçons, en constatant une prépondérance des activités dédiées aux garçons. Cette représentation étant plutôt caractéristique des animatrices intervenant auprès de cette tranche d'âge, cela tend à confirmer le constat de la fréquentation des structures d'animation davantage par les garçons que par les filles à l'adolescence. De la même façon que les animateurs, les animatrices tendent à proposer aux garçons et aux filles des activités conformes à leur genre. «Leurs hobbies, les gars, c'est plus le football, j'essaye de m'intéresser un peu au football pour discuter avec eux ; les filles heureusement, c'est plus des trucs de filles, les stars, les mecs, les fringues ».

\section{Des représentations du loisir différentes entre enfants et adolescent-e-s}

La CHD réalisée sur le corpus des discours des enfants et des adolescent-e-s a permis de dégager cinq profils différenciés. La variable âge, associée à chacune de ces classes, nous permet de repérer et de distinguer les représentations caractéristiques des enfants en accueil de loisirs (classes 2 et 5 ) et celles qui sont caractéristiques des adolescents en espace jeunes (classes 1, 3 et 4). La variable sexe, également associée, n'illustre pas toujours une classe ; toutefois, un tri croisé sur la variable sexe complète cette analyse et permet de distinguer le discours des enfants et des adolescent-e-s selon leur sexe.

\section{Des représentations du loisir entre jeu et activité pour les enfants}

Du côté des enfants, une première représentation (classe 2, $28 \%$ du discours, 115 UCE) envisage les loisirs d'un point de vue utilitariste. Les loisirs sont considérés comme un mode de garde sur un temps contraint pour l'enfant. En effet, son temps libre est ici organisé par les adultes puisque les enfants se rendent très majoritairement en centre de loisirs sur le temps de travail de leurs parents. «Les loisirs c'est comme des centres 
aérés et j'aime bien, les loisirs c'est des gens, des adultes qui gardent les enfants, ça sert les loisirs à garder les enfants, alors nos parents ils travaillent le mercredi, c'est pour nous occuper et nous garder ». Pour autant, les enfants considèrent aussi que les loisirs offrent un espace de liberté par la pratique du jeu, marquant sans doute, ici, leur appropriation et leurs marges de liberté dans cette organisation orchestrée par les adultes. «Les loisirs, c'est pour jouer, pour par exemple jouer au basket, jouer au foot, jouer à cache-cache, jouer à la gamelle, jouer avec les animateurs, jouer avec les copains quand ils viennent avec nous, dans le centre, ça sert à jouer ». Nous pouvons noter ici l'ambivalence de cette représentation des enfants où cohabitent contrainte sociale et espace de liberté. L'analyse de tris croisés révèle que si cette représentation est partagée de façon générale par les enfants, elle est toutefois davantage présente dans le discours des garçons.

Une deuxième représentation (classe 5, $28 \%$ du discours, 113 UCE) révèle les loisirs comme un lieu de réalisation personnelle et d'apprentissage par la pratique d'activités choisies par l'enfant, mais régulées et contrôlées par les adultes. L'enfant vient par choix en structure d'animation pour pratiquer des activités qui font sens pour lui et qui sont sources de réalisation de soi et de nouveaux apprentissages, le jeu n'en faisant pas partie. «Au centre, j'apprends à écouter, j'apprends à jouer, j'apprends à bricoler, à faire de la pêche dans les bois ». En référence à la classification de Passmore et French (2001), les enfants attribuent aux activités de loisirs une forme de productivité, intégrant une dimension de rentabilité «j'apprends des choses nouvelles » en opposition à la conception de gratuité des loisirs. «On peut aider les animateurs à faire des choses, par exemple le goûter, mais ce qui me plaît le plus c'est de faire des ateliers bois ». Les enfants distinguent ainsi les activités de loisirs, porteuses d'apprentissages, du jeu gratuit ; ce que confirme l'anti-profil qui écarte le jeu. L'analyse de tris croisés indique que cette seconde représentation est davantage caractéristique du discours des filles, ce qui montre que ces dernières envisagent davantage que les garçons les loisirs dans une perspective éducative. Par ailleurs, cette analyse souligne également que les garçons plébiscitent davantage des activités collectives, physiques et sportives, propices à l'activité ludique (selon le cadre) ; alors que les filles privilégient plutôt des activités individuelles, manuelles, de création et de découverte, débouchant le plus souvent sur la création d'un objet, témoin d'un résultat visible de l'activité.

\section{Des représentations du loisir entre autonomie et loisirs marchands pour les adolescent-e-s}

35 S'agissant des adolescent-e-s, ils et elles expriment à travers leurs représentations leurs attentes d'autonomie et de sociabilité entre pairs, et envisagent les loisirs dans une double finalité. D'une part, dans une finalité de convivialité (classe 3, $22 \%$ du discours, 89 UCE), qui s'étaye sur des discussions entre pairs et avec l'animateur. "Ici c'est l'endroit où on se retrouve, c'est le quartier général, il y a de quoi s'installer, on peut s'installer au chaud, on peut discuter tranquillement sans qu'il y ait les parents". D'autre part, dans une finalité de détente (classe 4, $7 \%$ du discours, 28 UCE), qui se traduit par des pratiques informelles de détente ou de défoulement. «Les loisirs, c'est des moments où tu t'amuses, où tu prends plaisir à faire quelque chose, ça sert à se détendre, à se défouler, à penser à autre chose, à se vider la tête par rapport à la vie de tous les jours, les cours ». Cette forme de loisirs s'étaye sur l'idée de liberté qu'offre ce 
type d'espace d'accueil en l'absence de contraintes ou d'engagement (par exemple, dans un projet ou une activité régulière demandant une forme d'assiduité).

Les loisirs dans le cadre de l'espace jeunes, c'est pas trop en termes d'activités, sauf de temps en temps, sinon en général quand je viens c'est distrayant, c'est un loisir parce que c'est distrayant mais on ne fait pas trop d'activités, on reste là. Je viens pour être avec les autres.

L'analyse des tris croisés montre que garçons et filles partagent ces représentations.

Par ailleurs, les adolescent-e-s envisagent également les loisirs (classe 1, 15\% du discours, 62 UCE) comme un espace de pratique d'activités, plutôt physiques, relevant $\mathrm{du}$ domaine marchand et comme l'occasion de participer à des séjours. "C'est l'animateur qui décide des activités et nous on choisit nos activités et il dit si c'est possible ou pas, il y a des loisirs comme le karting, le space laser et par exemple des nuitées ». Ces activités ou séjours s'inscrivent dans un programme élaboré par l'animateur en consultation avec les adolescent-e-s qui peuvent autofinancer ces activités.

Les activités, le programme, souvent les animateurs demandent qu'est-ce qui vous plairait et on dit, et après ils élaborent un programme et on s'inscrit.

Pour gagner de l'argent on peut faire des actions d'autofinancement, en fait à chaque fois on propose ce qu'on veut faire et ensuite on essaye de les mettre en place avec des actions d'autofinancement par exemple.

8 L'analyse de tris croisés selon le genre montre la préférence des adolescents pour des activités physiques et sportives (plutôt dans le domaine marchand), celle des adolescentes pour des activités favorisant la découverte et le lien social, et des projets s'inscrivant dans une démarche d'autofinancement.

L'anti-profil de ces trois classes montre que la plupart des adolescent-e-s s'accordent pour écarter de leurs loisirs le jeu et les activités de réalisation de soi de type loisir créatif, manuel, de plein air, souvent caractéristiques des accueils de loisirs dédiés aux enfants, qu'ils et elles considèrent, sans doute, comme des activités appartenant au domaine de l'enfance. Si les adolescent-e-s écartent le jeu de leurs loisirs, il semble qu'il s'agisse davantage d'exclure le terme « jeu » qui ferait davantage référence à l'enfance, que l'activité de jouer qui reste bien une forme intégrée à leur loisir, comme le prouvent les nombreux jeux - supports ou règles - qu'ils et elles évoquent : faire un babyfoot ou un billard, jouer sur l'ordinateur ou la console ou autre support numérique.

\section{Complexité et enjeux de la place du jeu}

En croisant les représentations des un-e-s et des autres, la question du jeu apparaît dans toute sa complexité dans ce champ de l'animation enfance et jeunesse. De plus, les discours se distinguent en fonction, d'une part, de l'âge des usagers et, d'autre part, du genre des usagers et des professionnel-le-s.

\section{Un jeu mis à la marge dans l'animation enfance}

Dans l'animation dédiée aux plus jeunes, nous pouvons identifier deux positionnements distincts sur la question du jeu. D'une part, une absence de considération pour le jeu en tant que loisir ou pur divertissement, au profit d'activités construites par les adultes 
pour les enfants en référence à leur évolution et à leur développement dans une perspective éducative. Si cette première orientation est partagée par l'ensemble des animateurs-trices, elle est néanmoins davantage caractéristique des animateurs. Ce résultat nous interpelle car, du côté des parents, a contrario, ce sont les mères, plus que les pères, qui envisagent le jeu dans une perspective éducative et développent des pratiques de jeu plus éducatives (Vincent, 2001). D’autre part, une considération du jeu comme une forme de loisir même si celle-ci reste secondaire par rapport à la forme plus contraignante des activités organisées par les adultes. Cette deuxième orientation est essentiellement développée par les animatrices qui valorisent, par ailleurs, davantage les dimensions affectives et sociales des loisirs.

Ces deux positionnements du jeu par rapport au loisir se retrouvent également chez les enfants qui envisagent, d'une part, le jeu comme vecteur de distraction et de loisir, de façon beaucoup plus marquée que les adultes d'ailleurs, d'autre part, comme vecteurs d'apprentissages au travers d'activités de réalisation de soi, notamment les filles. Concernant cette deuxième représentation, il est intéressant de noter que les enfants excluent le jeu libre de ces activités de réalisation de soi "où on leur fait faire", marquant une opposition entre réalisation de soi/apprentissage et jeu ; de cette façon, seules les activités dites " productives » permettraient la réalisation de soi (Constans et Gardair, 2018). Ainsi, les représentations des enfants rejoignent celles des adultes qui valorisent l'idée du « loisir éducatif» (Constans et Gardair, 2018) mais sans y inclure le jeu. Il apparait en corollaire que la seule finalité du jeu pour l'enfant serait de jouer. Dans ces contextes éducatifs, cette approche du jeu par défaut laisse à penser que la richesse heuristique, inhérente au cadre spécifique du jeu caractérisé par le second degré (Brougère, 2005 ; Bruner, 1983 ; Wallon, 1968) n'est ainsi perçue ni par ceux et celles qui s'y livrent (Brougère, 2003) ni par celles et ceux qui organisent les temps et activités.

\section{Une présence du jeu implicite dans l'animation jeunesse}

D'une manière générale, le terme «jeu " n'est pas usité concernant les accueils des adolescent-e-s, tant par les professionnel-le-s que par les adolescent-e-s eux-mêmes. Pour autant, ces différent-e-s acteurs-actrices n'excluent pas l'activité de jouer. Au contraire, ils et elles envisagent de nombreuses activités comme support de détente, dont certaines, peuvent être considérées comme des activités ludiques au regard des caractéristiques du jeu développées par Brougère (2005). Ces activités peuvent se présenter sous la forme de pratiques informelles (babyfoot, billard, jeu vidéo, etc.) ou s'inscrire dans un programme construit par l'adulte en concertation avec les adolescent-e-s. Dans ce cas, les activités plébiscitées par les jeunes et proposées par les animateurs-trices sont plutôt des activités physiques et sportives, se réalisant principalement dans le domaine marchand (karting, paintball, lasergame, etc.). Ainsi, pour les adolescent-e-s, davantage que pour les enfants, les pratiques de loisir peuvent être fondées sur la seule notion de détente (Dumazedier, 1988), laissant de fait une place au second degré, à la décision, à la frivolité et à l'incertitude. La quête de liberté et le besoin d'autonomie caractérisant souvent la période de l'adolescence, ainsi que le besoin de l'adolescent-e de se construire hors de la socialisation proposée par les adultes (Zaffran, 2000), peuvent trouver dans cette forme ludique de loisir un moyen de s'exprimer et d'affirmer une culture d'âge (Octobre, 2004; Octobre et Berthomier, 
2011), favorisant leur émancipation et leur construction identitaire (Erickson, 1968 ; Kindelberger, Le Floc'h et Clarisse, 2007).

Seuls les animateurs envisagent également une autre forme de loisir à l'adolescence, à visée éducative, axée plus particulièrement sur l'éducation à la citoyenneté par le biais d'activités dont la finalité est la production d'apprentissages, prioritairement dans l'espace social (apprentissage de règles sociales, formation du futur citoyen). Dans ce cas, comme chez les enfants, l'activité ludique peut être utilisée comme support d'apprentissage et, en ce sens, est détournée du pur divertissement.

\section{Des enjeux liés à la socialisation de genre}

Les animateurs, davantage que les animatrices, envisagent les loisirs dans une perspective éducative et valorisent les apprentissages et la performance. Ils ne considèrent pas (ou peu) le jeu dans les pratiques de loisirs (notamment chez les plus jeunes). De plus, ils s'adressent davantage aux adolescent-e-s, plus particulièrement aux garçons, auxquels ils proposent des activités, pour certaines ludiques, conformes à leur genre (activités physiques principalement). Ces résultats amènent notamment à interroger la place des activités physiques dans les activités ludiques durant l'enfance et à l'adolescence. A contrario des animateurs, les animatrices envisagent le jeu comme une forme de loisir, tant chez les enfants que chez les adolescent-e-s, auxquel-le-s elles proposent des activités ludiques différenciées et genrées. Elles privilégient aussi les aspects relationnels (communication, écoute, sécurité) dans leurs interactions avec les usagers. Les animateurs et les animatrices participent ainsi de façon spécifique au processus de socialisation de genre en opérant des distinctions entre filles et garçons à partir de leur conception des activités de loisirs. Ces résultats renvoient à ceux de Bessin (2009) qui montrent que même si les professionnel-le-s regrettent l'absence des filles dans les espaces de loisirs à l'adolescence, pour répondre aux envies de ceux qui les investissent (majoritairement des garçons), ils et elles reproduisent et renforcent les clivages de genre, et notamment les stéréotypes masculins, au travers des activités qu'ils et elles proposent. Les représentations et les pratiques des animateurs et des animatrices renvoient probablement à leur propre socialisation de genre et leur identité sexuée (Gallibour, 2006), à l'idéologie de la complémentarité des rôles sexués (Herman, 2006) et à une forme de reconnaissance sociale en répondant aux attentes de l'institution, des enfants, des adolescent-e-s et des parents (Bacou, 2010).

Ce contexte de loisirs propose ainsi aux enfants et aux adolescent-e-s des expériences de socialisation genrées. Cependant, dans le même temps, les filles et les garçons investissent de façon spécifique ce cadre structuré des loisirs. En effet, si les filles envisagent des activités ludiques dans le cadre de leurs loisirs, elles privilégient de manière plus marquée que les garçons, des activités sources d'apprentissages ou de lien social. Le jeu, sous ses différentes formes : jeu de rôles, construction de cabane, sport, babyfoot/billard, jeu vidéo, lasergame, etc., semble plus présent dans les loisirs organisés des garçons que dans ceux des filles, et ce quel que soit leur âge. Par ailleurs, les garçons privilégient davantage des activités de plein air, des activités physiques, les filles des activités de réalisation de soi, manuelles, de découverte. Ces différences entre les filles et les garçons dans les activités de loisirs sont bien documentées dans la littérature, que ce soit dans le cadre structuré des loisirs dans le champ de l'animation ou dans d'autres types de loisirs (Bradley et Inglis, 2012; Sharp et al., 2007). Comme 
dans d'autres milieux éducatifs (lieux d'accueil de la petite enfance, école, etc.), il est notable que la coprésence ne suffit pas pour créer de la mixité et de l'égalité entre les sexes (Céroux et Crepin, 2013 ; Rouyer, 2007), ce qui pose notamment la question de la déconstruction des stéréotypes sexués (Ayral et Raibaud, 2009; Bacou, 2010) et de la considération des processus de construction identitaire et de socialisation de genre (Rouyer, Croity-Belz et Prêteur, 2010 ; Rouyer, Mieyaa et Le Blanc, 2014).

\section{Conclusion}

En interrogeant la relation du jeu et de l'animation, on peut se demander si animer c'est introduire du jeu, donner une dimension ludique à une activité ou un projet, et si l'enfant, dans ce cadre, joue pour autant? Il semblerait que ce ne soit pas si simple. Les discours des enfants sont en effet marqués par une conception utilitariste des loisirs associée à la recherche de réalisation personnelle. Ceux des adolescents évoquent plus spécifiquement la recherche de convivialité et de détente. Du côté des adultes, la dimension éducative est très présente dans les représentations des animateurs-trices et cette conception est susceptible de réduire ou d'oblitérer les caractéristiques fondamentales du jeu (Brougère, 2005). Les analyses des discours des acteurs, tant du côté des publics que du côté des professionnel-le-s de l'animation, convergent avec les études antérieures (Brougère, 2005; Constans et Gardair, 2018; Gardair et Constans, 2011 ; Haglund 2015 ; Houssaye, 1995 ; Roucous, 2007) pour montrer que les loisirs des enfants restent largement investis par le projet éducatif et le contrôle des adultes. Dans cette perspective, le jeu est domestiqué, utilisé comme support d'apprentissages. En tant que pur divertissement, le jeu ne paraît pas trouver une place légitime au sein des loisirs organisés des enfants, qui semblent être soumis à un processus d'éducation permanente. Ce constat est d'autant plus paradoxal que le jeu semble être davantage autorisé à l'adolescence, par le biais de pratiques de loisirs fondées sur la seule détente, renvoyant ainsi à la conception du loisir à l'âge adulte (Dumazedier, 1998). Ce résultat est sans doute à mettre en lien avec la volonté d'associer loisir et éducation concernant les enfants, ainsi qu'avec la conception de l'enfant et de son statut par rapport à l'éducation. Considère-t-on l'enfant au présent ou comme un adulte en devenir ? Des recherches futures pourraient examiner plus directement cette question dans le champ de l'animation, mais également dans le champ familial et interroger les pratiques et les représentations du jeu et du loisir véhiculées par les parents en lien avec celles des animateurs-trices.

De plus, les analyses amènent à interroger les processus de socialisation de genre et de construction des identités sexuées à l'œuvre dans ce contexte des loisirs organisés. En effet, des différences repérées dans le discours des professionnel-le-s et des enfants et adolescent-e-s permettent d'éclairer certains des résultats mis en évidence dans notre étude. En particulier, les représentations, les pratiques des loisirs organisés et le cadrage de ceux-ci par les animateurs et animatrices semblent structurés par le genre dans un mouvement dialectique associant les adultes, les enfants et les adolescent-e-s. Sur la base de ces résultats, nous soulignerons la nécessité de poursuivre l'examen de la contribution des loisirs aux processus de socialisation de genre et de construction des identités sexuées, tant du côté des professionnel-le-s que des publics accueillis, en explorant notamment la contribution des représentations et pratiques éducatives des parents dans ceux-ci. 
n, dans une perspective inter-sectionnelle, il nous semble pertinent d'approfondir ces premiers résultats en considérant le jeu des rapports sociaux de classe dont nous n'avons pas pu tenir compte dans notre étude, mais qui constitue selon nous une perspective à même de mieux préciser les dynamiques complexes des représentations et pratiques des loisirs organisés, tant du côté des adultes que du côté des enfants et des adolescents.

\section{BIBLIOGRAPHIE}

ARTHENSTAEDT U., MIKULA G. \& BREDT C. (2009), « Gender role self-concept and leisure activities of adolescents », Sex Roles, 60, pp. 399-409.

AUGUSTIN J-P. \& GILLET J-C. (2000), L'animation professionnelle. Histoire, acteurs, enjeux, Paris, L'harmattan.

AYRAL S. \& RAIBAUD Y. (2014), Pour en finir avec la fabrique des garçons. Volume 2 : Loisirs, Sport, Culture, Pessac, Editions Maisons des Sciences de l'Homme d'Aquitaine.

BACOU M. (2010), Parcours sexués et processus de professionnalisation dans les métiers de l'animation en accueil de loisirs, thèse de doctorat, Université Toulouse 2.

BARRERE A. (2011), L'éducation buissonnière. Quand les adolescents se forment par eux-mêmes, Paris, Armand Colin.

BARTKO W.T. \& ECCLES J.S. (2003), « Adolescent participation in structured and unstructured activities : a person-oriented analysis », Journal of Youth and Adolescence, 32(4), pp. 233-241.

BESSIN M. (2009), « Focus : la division sexuée du travail social », Informations Sociales, 152, pp. 70-73.

BOYER R., BOUNOURE A. \& DELCLAUX M. (1991), Paroles de lycéens. Les études, les loisirs, l'avenir, Paris, Éditions de l'Institut National de Recherche Pédagogique.

BRADLEY G.L. \& INGLIS B.C. (2012), « Adolescent leisure dimensions, psychosocial adjustment, and gender effects ", Journal of Adolescence, 35, pp. 1167-1176.

BRONFENBRENNER U. (1979), The ecology of human development, Cambridge, Harvard University Press.

BROUGERE G. (1995), Jeu et éducation, Paris, L'harmattan.

BROUGERE G. (2003), « Le jeu entre éducation et divertissement », Médiation et Information, 18, pp. 43-52.

BROUGERE G. (2005), Jouer/apprendre, Paris, Economica.

BROUGERE G. \& BEZILLE H. (2007), « De l'usage de la notion d'informel dans le champ de l'éducation », Revue Française de Pédagogie, 158, pp. 117-160.

BRUNER J.S. (1983), Le développement de l'enfant : savoir faire, savoir dire, Paris, Presses Universitaires de France. 
CEROUX B. \& CREPIN C. (2013), « Rapport aux loisirs et pratiques des adolescents », Politiques Sociales et Familiales, 111, pp. 59-64.

CONSTANS S. \& GARDAIR E. (2018), « Représentations et pratiques des loisirs des enfants et adolescents : le paradoxe de la dimension éducative des loisirs », Bulletin de Psychologie, 554, tome 71(2), pp. 563-578.

COURT M. (2010), Corps de filles, corps de garçons : une construction sociale, Paris, La Dispute.

DAVISSE A. (2006), « Filles et garçons dans les activités physiques et sportives : de grands changements et de fortes permanences ", in A. Dafflon-Novelle (dir.), Filles-garçons. Socialisation différenciée ?, Grenoble, Presses Universitaires de Grenoble, pp. 287-301.

DAVISSE A. \& LOUVEAU C. (1998), Sports, école, société. La différence des sexes, Paris, L'Harmattan.

DELALANDE J. (2001), La cour de récréation. Pour une anthropologie de l'enfance, Rennes, Presses Universitaires de Rennes.

DUMAZEDIER J. (1988), Révolution culturelle du temps libre : 1968-1988, Paris, Méridiens-Klincksieck.

ERICKSON E. (1968), Enfance et société, Paris, Delachaux et Niestlé.

FREDRICKS J.A. \& ECCLES J.S. (2008), « Participation in extracurricular activities in the middle school years : are there developmental benefits for African American and European American youth? ", Journal of Youth and Adolescence, 37(9), pp. 1029-1043.

GALLIBOUR E. (2006), « Point de vue sur l'égalité des sexes dans le champ de l'animation bénévole et professionnelle », in J-C. Gillet \& Y. Raibaud (dirs.), Mixité, parité, genre dans les métiers de l'animation, Paris, L'Harmattan, pp. 159-170.

GARDAIR E. \& CONSTANS S. (2011), « Représentations de la prise en charge des enfants et des adolescents en contexte de loisirs chez des animateurs socioculturels ", Approche Neuropsychologique des Apprentissages chez l'Enfant, 112/113(23), tomes II et III, pp. 201-207.

GILLET J.-C. \& RAIBAUD Y. (2006), Mixité, parité, genre dans les métiers de l'animation, Paris, L'Harmattan.

HAGLUND B. (2015), « Pupil's opportunities to influence activities : a study of everyday practice at a Swedish leisure-time centre », Early Child Development and Care, 185(10), pp. 1556-1568.

HERMAN E. (2006), « Le genre en centre de loisirs : faire avec et "mettre de soi" ", in J-C. Gillet \& Y. Raibaud (dirs.), Mixité, parité, genre dans les métiers de l'animation, Paris, L'Harmattan, pp. 87-101. HERMAN E. (2007), « La bonne distance. L'idéologie de la complémentarité légitimée en centres de loisir », Cahiers du Genre, 42, pp. 121-139.

HOUSSAYE J. (1995), Et pourquoi que les colos, elles sont pas comme ça? Histoires d'ailleurs et d'Asnelles, Paris, Matrice.

HOUSSAYE J. (1998), « Le centre de vacances et de loisirs prisonnier de la forme scolaire », Revue Française de Pédagogie, 125, pp. 95-107.

KINDELBERGER C., LE FLOC'H N. \& CLARISSE R. (2007), « Les activités de loisirs des enfants et des adolescents comme milieu de développement », L'Orientation Scolaire et Professionnelle, 36(4), pp. 485-502.

LE FLOC'H N. (2005), Approche écosystémique des rythmicités attentionnelles de l'enfant. Étude des effets des aménagements du temps scolaire, des loisirs collectifs et du travail parental sur les niveaux et les variations de l'attention de l'enfant de 5 à 10 ans, thèse de doctorat, Université de Tours. 
LEVERSEN I., DANIELSEN A.G., BIRKELAND M.S. \& SAMDAL O. (2012), « Basic psychological need satisfaction in leisure activities and adolescents' life satisfaction », Journal of Youth and Adolescence, 41(12), pp. 1588-1599.

LILLARD A.S. (2015), « Development of play », in L.S. Liben, U. Muller \& R.M Lerner. (eds.), Handbook of child psychology and developmental science : cognitive processes, New York, John Wiley \& Sons Inc, pp. 425-468.

MAHONEY J.L., LARSON R.W., ECCLES J.S. \& LORD H. (2005), « Organized activities as development contexts for children and adolescents ", in J.L. Mahoney, R.W. Larson \& J.S. Eccles (eds.), Organized activities as contexts of development : extracurricular activities, after-school, and community programs, Mahwah, Lawrence Erlbaum, pp. 3-22.

MARUEJOULS E. (2011), « La mixité à l'épreuve des loisirs des jeunes dans trois communes de Gironde », Agora Débats/Jeunesses, 59(3), pp. 79-91.

MCHALE S.M., KIM J.-Y., WHITEMAN S. \& CROUTER A.C. (2004), « Links between sex-typed time use in middle childhood and gender development in early adolescence », Developmental psychology, 40, pp. 868-881.

MERCIER-LEFEVRE B. (2014), « Fabriquer du masculin dans les formations en sciences et techniques des activités physiques et sportives (STAPS) ? ", in S. Ayral \& Y. Raibaud (eds.), Pour en finir avec la fabrique des garçons, volume 2, Pessac, Maison des Sciences de l'Homme d'Aquitaine, pp. 73-91.

MOLLO-BOUVIER S. (1994), « De l'école aux vacances. Prolégomènes à une analyse sociologique des vacances des enfants ", Revue Française de Pédagogie, 106, pp. 79-90.

OCTOBRE S. (2004), Les loisirs culturels des 6-14 ans, Paris, La Documentation Française.

OCTOBRE S. \& BERTHOMIER N. (2011), « L'enfance des loisirs. Éléments de synthèse », Culture Etudes, 6, pp. 1-12.

PASSMORE A.E. \& FRENCH D. (2001), « Development and administration of a measure to assess adolescent's participation in leisure activities ", Adolescence, 36(141), pp. 67-75.

RAIBAUD Y. (2007), Genre et loisirs des jeunes, Empan, 65, pp. 67-73.

RAIBAUD Y. (2014). «Introduction Sport, culture, loisirs : ces autres lieux de production et de consolidation de l'identité masculine, in S. Ayral \& Y. Raibaud (eds.), Pour en finir avec la fabrique des garçons ", volume 2, Pessac, Maison des Sciences de l'Homme d'Aquitaine, pp. 9-25.

REINERT M. (1983), « Une méthode de classification descendante hiérarchique : application à l'analyse lexicale par contexte », Les cahiers de l'analyse des données, VIII, 2, pp. 187-198. REINERT M. (1993), « Les mondes lexicaux et leur logique à travers l'analyse statistique d'un corpus de récits de cauchemars », Langage et société, 66, pp. 5-39.

ROUCOUS N. (2007), « Les loisirs de l'enfant ou le défi de l'éducation informelle », Revue Française de Pédagogie, 160, pp. 63-73.

ROUYER V. (2007), La construction de l'identité sexuée, Paris, Armand Colin.

ROUYER V., CROITY-BELZ S. \& PRÊTEUR Y. (2010), Genre et socialisation de l'enfance à l'âge adulte. Expliquer les différences, penser l'égalité, Toulouse, Erès.

ROUYER V., MIEYAA Y. \& LE BLANC A. (2014), « Socialisation de genre et construction des identités sexuées. Contextes sociétal et scientifique, acquis de la recherche et implications pratiques. Note de synthèse ", Revue française de pédagogie, n 187, pp. 97-137. 
SHARP E.H., COATSWORTH J.D., DARLING N., CUMSILLE P. \& RANIERI S. (2007), « Gender differences in the self-defining activities and identity experiences of adolescents and emerging adults ", Journal of Adolescence, 30, pp. 251-269.

SIMPKINS S.D., RIPKE M., HUSTON A.C. \& ECCLES J.S. (2005), « Predicting participation and outcomes in out-of-school activities : Similarities and differences across social ecologies ", New Directions for Youth Development, 105, pp. 51-69.

SMITH C., SANTUCCI D., XU S., COX A. \& HENDERSON K.A. (2012), « "I love my job, but..." : A narrative analysis of women's perceptions of their careers in parks and recreation ", Journal of Leisure Research, 44(1), pp. 52-69.

VANDELL D.L., LARSON R.W., MAHONEY J.L. \& WATTS T.W. (2015), « Children's organized activities ", in M.H. Bornstein, T. Leventhal \& R.M. Lerner (eds.), Handbook of child psychology and developmental science : ecological settings and processes, New York, John Wiley \& Sons Inc, pp. 305-344.

VINCENT S. (2001), Le jouet et ses usages sociaux, Paris, La Dispute.

WALLON H. (1968), L'évolution psychologique de l'enfant, Paris, Armand Colin.

WINNYKAMEN F. (2009), Les relations sociales chez l'enfant : genèse, développement, fonctions, Paris, Armand Colin.

YONNET P. (1999), Travail, loisir. Temps libre et lien social, Paris, Gallimard.

ZAFFRAN J. (2000), Les collégiens, l'école et le temps libre, Paris, Syros.

\section{NOTES}

1. Distinct du jouet qui « suppose une relation à l'enfance et une ouverture, une indétermination quant à l'usage, c'est-à-dire l'absence de relation directe avec un système de règles qui organiserait son utilisation » (Brougère, 1995, p. 14).

2. Travail professionnel ou scolaire et astreintes s'y rattachant.

3. Obligations sociales, administratives, familiales et domestiques.

4. Moyenne 7,5; Ecart type 1,43. Seuls 2 enfants sont âgés de 5 ans.

5. Moyenne 16,10 ; Ecart type 2,88. Seuls 4 adolescents ont moins de 15 ans.

6. En raison de la confidentialité des données, nous n'avons eu accès aux données socioéconomiques uniquement de façon globale, ce qui ne permet pas une comparaison intragroupe à partir de cette variable.

7. Une quatrième représentation caractéristique du discours des animatrices (classe $4,20 \% \mathrm{du}$ discours, 424 UCE) souligne le fonctionnement et l'importance de la dimension partenariale des structures de loisirs.

\section{RÉSUMÉS}

Cet article a pour but d'interroger la place du jeu dans les loisirs organisés des enfants et des adolescent-e-s dans le cadre de structures d'animation, à travers l'examen des représentations 
des loisirs des animateurs-trices, des enfants et des adolescent-e-s. Par le biais d'entretiens semidirectifs, nous avons recueilli le point de vue de 80 animateurs-trices et de 50 enfants et adolescent-e-s usagers d'un accueil de loisirs sans hébergement ou d'un espace jeunes. Nos résultats soulignent la complexité de la place du jeu et la nécessité d'interroger les enjeux liés à la socialisation de genre des enfants et des adolescent-e-s, dans les loisirs organisés dans le champ de l'animation enfance et jeunesse.

This article addresses the place of play in the children's and teenagers structured leisure activities in leisure centers, considering the representations of leisure from leisure activities leaders, children and teenagers. We conducted semi-directed interviews with 80 professionals, and 50 children and young people attending a leisure center during the day. Our results highlighted the complexity of the place of play and the necessity to take into account the issues linked to gender socialization of children and teenagers in the field of structured youth leisure activities.

\section{INDEX}

Mots-clés : jeu, loisir, structure d'animation, représentation, socialisation de genre, enfance, adolescence

Keywords : play, leisure, leisure center, representation, gender socialization, childhood, adolescence

\section{AUTEURS}

\section{STÉPHANIE CONSTANS}

Centre de Recherche en Education de Nantes (EA 2661), Université de Rennes 1

\section{VÉRONIQUE ROUYER}

Laboratoire de psychologie (EA 4139), Université de Bordeaux

\section{EMMANUÈLE GARDAIR}

Laboratoire de Psychologie des Pays de la Loire (EA 4638), Université de Reims Champagne Ardenne 